\title{
Rapidly rotating strange stars
}

\author{
D. Gondek-Rosińska, P. Haensel, J. L. Zdunik
}

Copernicus Astronomical Center, Bartycka 18, Warszawa, Poland

E. Gourgoulhon

DARC, CNRS, Observatoire de Paris, F-92195 Meudon Cedex, France

\begin{abstract}
We study effects of the strange quark mass, $m_{s}$, and of the QCD interactions, calculated to lowest order in $\alpha_{c}$, on the rapid rotation of strange stars (SS). The influence of rotation on global parameters of $\mathrm{SS}$ is greater than in the case of the neutron stars (NS). We show that independently of $m_{s}$ and $\alpha_{c}$ the ratio of the rotational kinetic energy to the absolute value of the gravitational potential energy $T / W$ for a rotating SS is significantly higher than for an ordinary NS. This might indicate that rapidly rotating SS could be important sources of gravitational waves.
\end{abstract}

Recently, it has been shown that SS are not a subject to the r-mode instability (Madsen 1998, Stergioulas 1998) in a contrast to newly born NS. Therefore SS formed in a collapse of rotating stellar cores can rotate very fast. We have calculated exact models of the uniformly rotating SS using multi-domain spectral methods, used previously for calculating rapidly rotating SS models with $m_{s}=\alpha_{\mathrm{c}}=0$ (Gourgoulhon et al.,1999, A\&A 349, 851). We construct $M-R$ curves for SSs rotating with a broad range of angular velocity and calculate the ratio $T / W$, relevant for stars stability with respect to triaxial deformations. We present results for two EOS of strange quark matter, both with Bag Constant $B=60 \mathrm{MeV} / \mathrm{fm}^{3}$ : SQ1 with $m_{s}=200 \mathrm{MeV} / \mathrm{c}^{2}, \alpha_{\mathrm{c}}=0$, and SQ2 with $m_{s}=200 \mathrm{MeV} / \mathrm{c}^{2}, \alpha_{\mathrm{c}}=0.6$ and compared them with SQ0 EOS with $m_{s}=\alpha_{c}=0$. Results for different values of $B$ can be obtained from those for $B=60 \mathrm{MeV} / \mathrm{fm}^{3}$ using scaling properties of corresponding quantities with respect to the change of $B$. The main conclusions can be summarised as follows:

- There is a large increase of the maximum mass and corresponding radius of SS as the rotation rate increases from zero to the equatorian mass-shedding limit (Fig. 1a). The rotation increases the maximum allowable mass of SS by $\sim 40 \%$ compared to $\sim 20 \%$ for NS and the equatorial radius by $\sim 50 \%$ compared to $\sim 30 \%$ for NS. At fixed $B$ the maximum mass is for SQ0 model rotating with Keplerian velocity (the maximum mass $M=2.861 M_{\odot}$ is for the lowest allowable $\left.B=58.9 \mathrm{MeV} / \mathrm{fm}^{3}\right)$.

- At fixed $B$, the effect of $m_{s}$ on rotating SS models is much larger than that of $\alpha_{\mathrm{c}}$. Both decreases stellar mass and radius (Fig. 1a, 1b). The M-R curves for sequences of SS rotating with high fixed velocity $f=\Omega / 2 \pi$ reminds $\mathrm{M}(\mathrm{R})$ relations for ordinary NS. When $f$ increases the $M(R)$ curves for all models are more and more flat. Fast rotation causes a large increase of a radius of SS especially for small masses. 

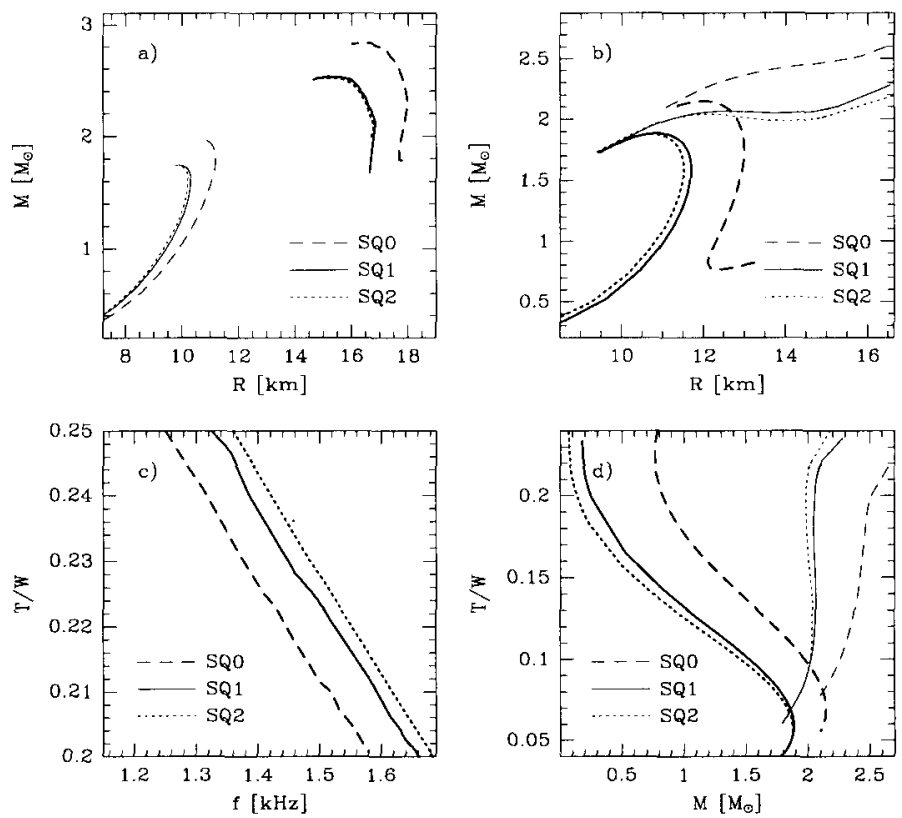

Figure 1. a) Mass vs. radius for maximally rotating (thick lines) and static (thin lines) SS. b) Mass vs. radius for sequences of SS rotating with frequency $f=1.1 \mathrm{kHz}$ (thick lines) and $f=1.4 \mathrm{kHz}$ (thin lines). c) Kinetic to gravitational energy ratio vs. the rotation frequency for supramassive SS rotating with Keplerian velocity. d) $T / W$ vs. mass for SS rotating with fixed $f=1.1$ and $1.4 \mathrm{kHz}$ (thick and thin lines, respectively). The ratio $T / W$ increases along each sequence as central density decreases.

- The values of $T / W$ for SS rotating at the Keplerian velocity can significantly exceed 0.2 (see Fig. 1c for supramassive stars). There are much greater than for NS (more that twice). All this seems to indicate that triaxial instabilities could develop more easily in rotating SS than in NS. The value of $T / W$ increases with decreasing stellar mass for all considered models of SS. We have $M=2.82,2.53,2.52, M_{\odot}$ for $T / W=0.2$ and $M=2.14,2.01,1.99 M_{\odot}$ for $T / W=0.25$ for $\mathrm{SQ} 0, \mathrm{SQ} 1, \mathrm{SQ} 2$ respectively.

- Unusually high ratio $T / W$ is characteristic not exclusively for supramassive stars but can be reached also for rapidly rotating SS with small and moderate masses (Fig. 1d). For stars rotating with fixed frequency, $T / W$ increases with decreasing central density. The large value of $T / W$ results from a flat density profile combined with strong equatorial flattening of rapidly rotating SS.

Acknowledgments. This work was supported in part by the following grants KBN-2P03D01413 and KBN-2P03D02117. 